\title{
Dependencia térmica del proceso de inversión de la imanación en microhilos magnéticos biestables
}

\author{
M. VÁZQUEZ, A.P. ZHUKOV, K.L. GARCÍA, K.R. PIROTA, R. VARGA,J.L. MARTÍNEZ \\ Instituto de Ciencia de Materiales, CSIC. 28049 Madrid
}

\begin{abstract}
En este trabajo se resumen los más recientes resultados alcanzados relativos al comportamiento magnético de microhilos amorfos ricos en Fe cubiertos de capa aislante en función de la temperatura de medida (en el rango de 5 a $300 \mathrm{~K}$ ). Estos microhilos, debido a su elevada magnetostricción, presentan comportamiento magnético biestable cuando su longitud supera un valor crítico determinado por la intensidad de la anisotropía magnética axial. Esta anisotropía depende en particular del espesor de la cubierta protectora de Pyrex, de modo que al reducir la temperatura se inducen fuertes tensiones mecánicas (hasta $400 \mathrm{MPa}$ ) como consecuencia de la diferencia de valor del coeficiente de expansión térmica con el núcleo metálico. Se comprueba asímismo que el modo de sujección del microhilo al portamuestras puede generar fuertes tensiones de compresión y dar lugar a la desaparición de comportamiento biestable.
\end{abstract}

Palabras Clave.- Microhilos Magnéticos Amorfos, Inversión de la Imanación, Dependencia con la Temperatura, Biestabilidad Magnética

\section{Magnetization reversal process in bistable microwires and its temperature dependence}

The temperature dependence of the magnetic behaviour of Fe-based glass-coated amorphous microwires with positive magnetostriction has been studied in the temperature range from 5 to $300 \mathrm{~K}$. For long enough microwires, hysteresis loops are squared shaped in the whole temperature range denoting the existence of a large domain with axial easy axis. For short microwires, magnetic bistability is partially lost near room temperature but may be recovered at low temperature. This is interpreted considering the influence of the shape anisotropy: in short wires the demagnetizing field may become large enough to destroy the single domain structure. The influence of coating is also considered by studying microwires with different coating thickness. Transverse magnetoelastic anisotropy up to around 400 MPa can be induced at low temperature as a consequence of the difference in thermal expansion coefficients between metallic nucleus and insulating coat. Additional changes in the low-temperature loops have been identified to be a consequence of the way how the microwires are fixed to the sample's holder. Under particular circumstances transverse anisotropy induced at low temperature may destroy the bistable behavior.

Key Words.- Magnetic Amorphous Microwires, Magnetization Reversal, Temperature Dependence, Magnetic Bistability

\section{INTRODUCCIÓN Y ASPECTOS EXPERIMENTALES}

El reciente interés mostrado en hilos magnéticos con diámetro del orden de micras preparados por técnicas de solidificación ultrarápida se debe a sus excelentes propiedades magnéticas lo que les convierte en buenos candidatos para ser empleados como elementos sensores en distintos dispositivos [1,2]. En particular, mediante la técnica de enfriamiento ultrarápido y estiramiento se pueden fabricar microhilos magnéticos con estructura amorfa recubiertos por una capa aislante de vidrio Pyrex. Su diámetro típico es entre 1 y 30 micras, mientras que la cubierta de vidrio varía entre 2 y 15 micras.

Entre estos microhilos, los que poseen composición rica en Fe poseen elevada constante de magnetostricción (del orden de 30 p.p.m.) lo que les confiere un eje de fácil imanación axial debido a la anisotropía magnetoelástica introducida por el proceso de fabricación. Esto da lugar a la existencia de una estructura magnética monodominio en el cual, la inversión de la imanación se produce mediante un único salto Barkhausen que da lugar a comportamiento magnético biestable (ciclo de histeresis axial de forma cuadrada). Este salto es consecuencia del desenganche y propagación de una única pared a lo largo del hilo [3].

En trabajos previos se estudió el comportamiento magnético biestable en muestras de diferentes características geométricas [4], teniendo en cuenta la influencia de las tensiones mecánicas [5], o bien la temperatura de medida [6]. En este trabajo, se resumen algunos de los resultados más representativos obtenidos recientemente teniendo en cuenta la influencia de factores como la longitud (anisotropía de forma), y la relación entre el diámtro metálico y el total (anisotropía magnetoelástica).

Los microhilos amorfos recubiertos de capa aislante se preparan por la técnica de Taylor-Ulitovski [1]. En este trabajo se presentan resultados para microhilos de composición rica en $\mathrm{Fe}\left(\mathrm{Fe}_{72.5} \mathrm{Si}_{12.5} \mathrm{~B}_{15^{\prime}}\right.$ $\mathrm{Fe}_{775} \mathrm{Si}_{75} \mathrm{~B}_{15}$ y $\mathrm{Fe}_{70} \mathrm{Si}_{10} \mathrm{~B}_{15} \mathrm{C}_{5}$ ) caracterizados por su relativamente elevada magnetostricción (entorno a 20-30 p.p.m.). La longitud de los microhilos estudiados ha sido entre 1.5 y $15 \mathrm{~mm}$, mientras que sus diámetros metálico y total ha variado según los casos entre 3 y 30 micras, respectivamente, con objeto de estudiar microhilos que posean diferente relación, $\rho=\mathrm{d}_{\text {met }} / \mathrm{d}_{\mathrm{to}{ }^{\prime}}$ diámetro metálico, $\mathrm{d}_{\text {met' }}$ a diámetro total, $\mathrm{d}_{\mathrm{tot}}$.

Las medidas magnéticas y en particular los ciclos de histéresis han sido realizados en un magnetómetro SQUID en el rango de temperaturas entre 5 y $300 \mathrm{~K}$. El campo remanente de las bobinas ha sido cuidadosamente minimizado para reducir la asimetría de los ciclos. Asimismo, se ha puesto especial atención en el modo de fijar los microhilos al portamuestras. En concreto, se han empleado dos métodos que dan lugar a resultados diferentes cuya interpretación ha sido de mucha importancia. 


\section{RESULTADOS EXPERIMENTALES}

\subsection{Microhilos de diferente longitud}

Como es sabido, la longitud de las muestras es un parámetro muy importante a la hora de determinar el proceso de inversión de la imanación en los hilos biestables. En hilos de suficiente longitud, en la zona central aparece un único dominio magnético axial, y en los extremos se forman pequeñas estructuras de dominios de cierre para minimizar la energía magnetostática. Estas estructuras de dominios de cierre tienen una longitud finita que depende de las características del hilo y en particular de la intensidad de la anisotropía magnética axial [7,3]. Así, en hilos de corta longitud no se observa biestabilidad debido al solapamiento de estas estructuras de cierre provenientes de los dos extremos del hilo con lo que desaparece el monodominio central. En el caso de los microhilos ricos en Fe estudiados en este trabajo, la longitud de los dominios de cierre es del orden de $1 \mathrm{~mm}$, y por lo tanto la longitud crítica para observar biestabilidad (a temperatura ambiente) es del orden de $2 \mathrm{~mm}$.

En la Figura 1a se muestra el ciclo de histéresis medido a diferentes temperaturas para un microhilo de composición $\mathrm{Fe}_{89} \mathrm{Si}_{1} \mathrm{~B}_{1} \mathrm{C}_{3} \mathrm{Mn}_{4}$ y 10 $\mathrm{mm}$ de longitud. Como se puede apreciar, el ciclo de histéresis es biestable en todo el rango de temperaturas. En la Figura 1 b, se muestran los ciclos de histéresis para un hilo de iguales características salvo que su longitud es de $2 \mathrm{~mm}$. En este caso, los ciclos son rectangulares a bajas temperaturas mientras que a temperaturas próximas a la ambiente se pierde el comportamiento biestable (el proceso de inversión de la imanación se lleva a cabo en 2 grandes saltos Barkhausen). En la Figura 1c se representa la dependencia térmica del campo coercitivo o de inversión de la imanación para las dos muestras de diferente longitud.

\subsection{Microhilos de diferentes diámetro}

En las figuras $2 \mathrm{a}$ y $2 \mathrm{~b}$ se representan los ciclos de histéresis para dos microhilos de igual longitud (10 mm), diámetro total (23,0 micras) y composición ( $\mathrm{Fe}_{70} \mathrm{~B}_{15} \mathrm{Si}_{10} \mathrm{C}_{5}$ ), pero distintos diámetros de núcleo metálico (3.7 y 10.8 micras, respectivamente). En la figura 3c se representa la dependencia térmica del campo coercitivo para los dos microhilos. Como se puede observar el comportamiento biestable se mantiene ahora en ambos casos en todo el rango de temperaturas, y el campo coercitivo del microhilo de diámetro metálico inferior presenta valores más elevados.

Como ha sido estudiado previamente [1,2], el proceso de fabricación induce notables tensiones mecánicas en estos microhilos: tensiones de origen térmico debido al brusco enfriamiento, tensiones de tracción por estiramiento durante el proceso, y por último debido a los diferentes coeficientes de expansión térmica de los componentes del microhilo. La intensidad de las tensiones, y las correspondientes densidades de energía magnetoelásticas, es mayor en aquellos hilos con mayor cubierta aislante lo que da lugar a la mayor coercitividad del microhilo de 3,7 micras de diámetro metálico. Por otra parte, debido a los diferentes coeficientes de expansión térmica del núcleo y de la cubierta, al reducir la temperatura aparecen tensiones adicionales que contribuyen a incrementar la coercitividad como se analiza en lo que sigue.

\section{ANALISIS DE LOS RESULTADOS}

\subsection{El campo de anisotropía axial efectivo y su dependencia térmica}

El comportamiento biestable magnético aparece como consecuencia de la existencia de un dominio suficientemente grande donde el eje de fácil imanación es axial, para lo cual es preciso que exista una anisotropía axial magnética suficientemente intensa. De forma general,
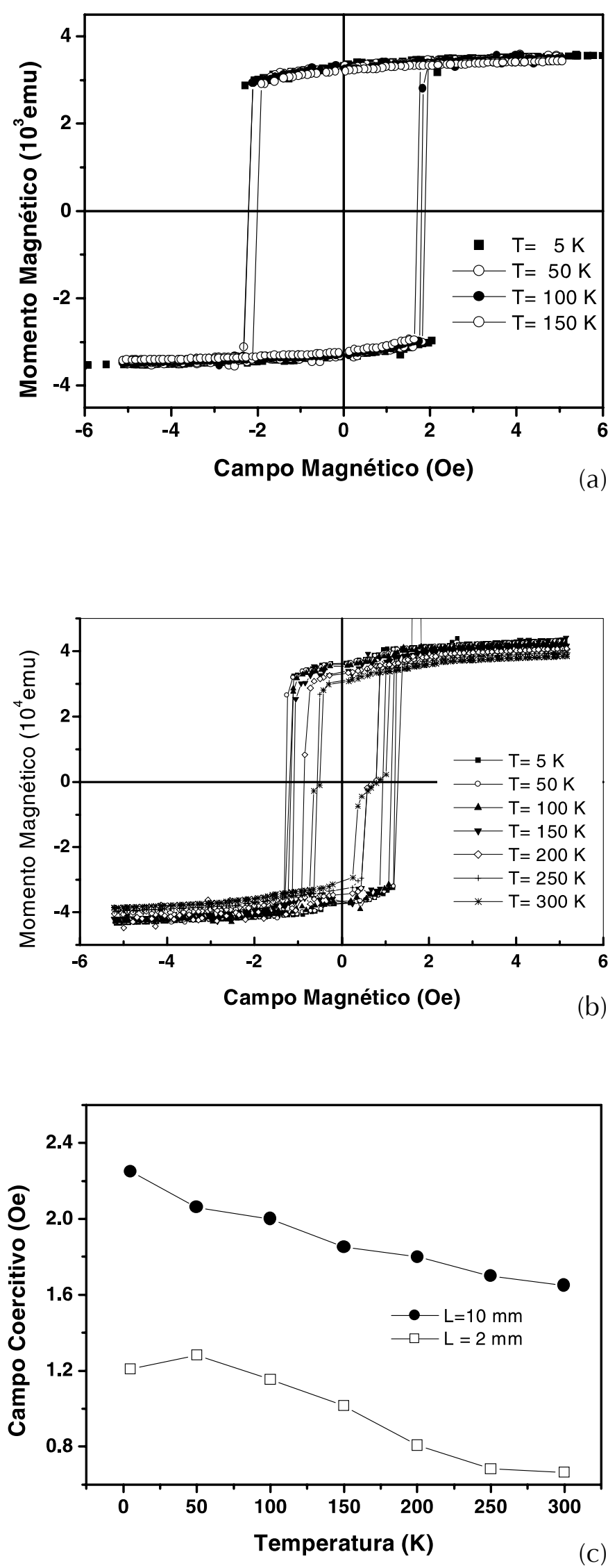

Figura 1.- Dependencia térmica del ciclo de histéresis de un microhilo de composición $\mathrm{Fe}_{89} \mathrm{Si}_{1} \mathrm{~B}_{1} \mathrm{C}_{3} \mathrm{Mn}_{4}$ y longitud $2 \mathrm{~mm}$ (a) y $10 \mathrm{~mm}$ (b). La evolución del campo coercitivo se indica en (c). 


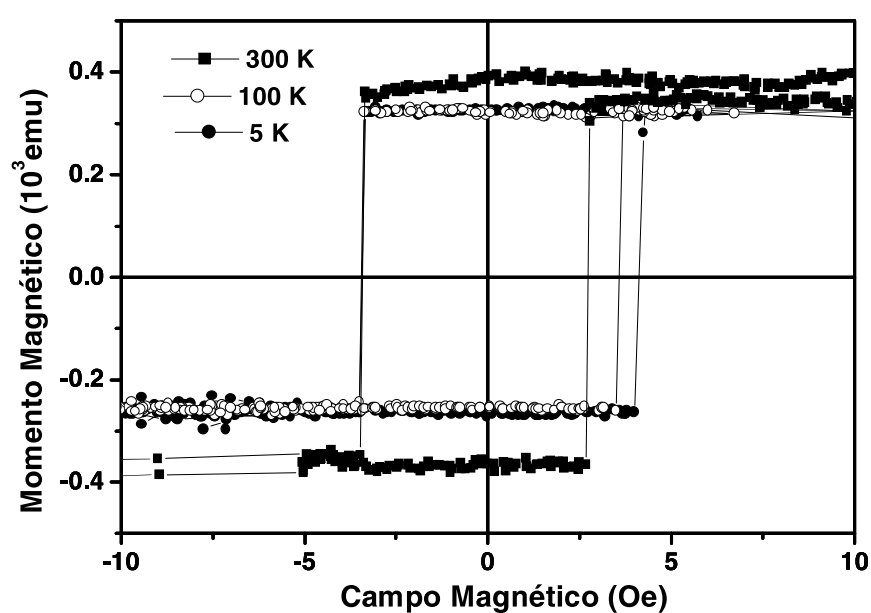

(a)
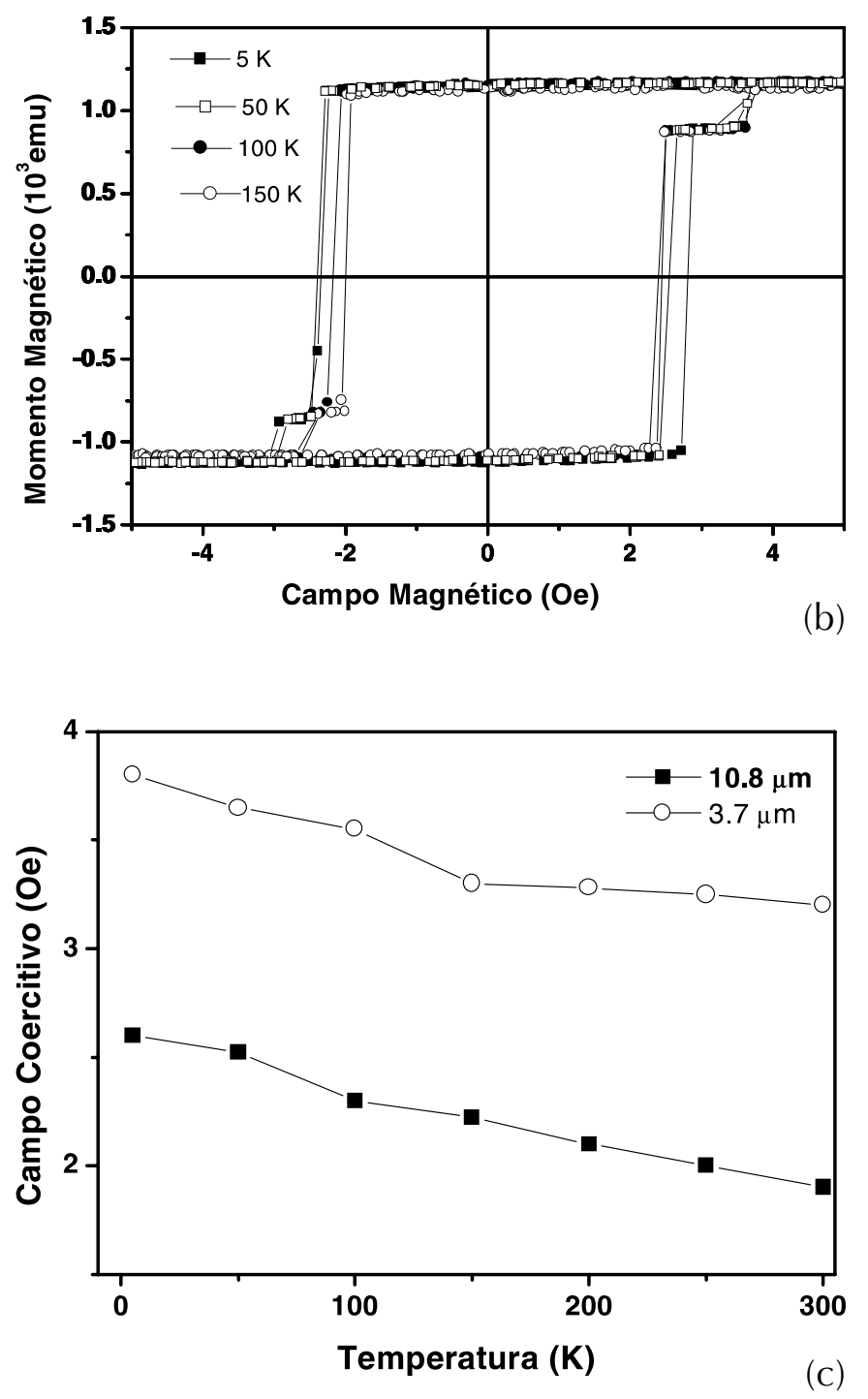

Figura 2.- Dependencia térmica de los ciclos de histérsis de un microhilo de composición $\mathrm{Fe}_{70} \mathrm{~B}_{15} \mathrm{Si}_{10} \mathrm{C}_{5}$, longitud $10 \mathrm{~mm}$, diámetro total 20 micras, y diámetro metálico 3.7 (a), y 10.8 (b) micras. Las correspondientes variaciones del campo coercitivo se indican en (c). el campo de anisotropía efectivo para nuestros microhilos se puede expresar como:

$$
H_{k}^{e f f}=H_{k}+N M_{s}
$$

al cual contribuyen el campo de anisotropía magnetoelástica, $H_{k^{\prime}}$ y el de anisotropia de forma, $H_{s h}$.

En relación al campo de anisotropía de forma, $H_{s h}=N M_{s}(T)$, el factor desimanador axial, $N$ (del orden de $10^{-5}$ para las dimensiones geométricas consideradas), depende exclusivamente de la geometría y por lo tanto no varía con la temperatura, mientras que la imanación de saturación, $M_{s^{\prime}}$ aumenta al disminuir la temperatura. Por lo tanto, el campo de anisotropía de forma disminuye con la temperatura disminuyendo así el campo de anisotropía axial efectivo.

Por otra parte, la dependencia térmica del campo de anisotropía magnetoelástico se puede expresar como:

$$
H(T)_{k}=\frac{3 \lambda(T) \sigma(T)}{\mu_{0} M_{s}(T)}
$$

A esta dependencia térmica contribuyen 3 factores: i) la mencionada dependencia de la imanación de saturación, $M_{s}(T)$, ii) la dependencia térmica de la magnetostricción, que se puede expresar como [8]:

$$
\lambda(T)=\alpha^{*}\left(\mu_{0} M_{s}(T)\right)^{3}
$$

y iii) la dependencia térmica de las tensiones internas, $\sigma_{\text {thexp. }}(T)$, que en este caso surge como consecuencia de las deformaciones debidas a los diferentes coeficientes de expansión térmica del núcleo metálico y la capa aislante [9]. Esta deformación se puede expresar como $\varepsilon=\left(\alpha_{g}\right.$ $\left.-\alpha_{\mathrm{m}}\right) \Delta \mathrm{T}$, siendo $\left(\alpha_{\mathrm{g}}-\alpha_{\mathrm{m}}\right)$ la diferencia entre coeficientes de expansión térmica de la capa aislante y el núcleo metálico, y $\Delta \mathrm{T}$ el incremento de temperatura. Las tensiones asociadas a esta deformación son complejas y presentan componentes de carácter axial, $\sigma_{z z^{\prime}}$ radial, $\sigma_{\mathrm{r}^{\prime}}$ y circular, $\sigma_{\phi \phi}[5]$ :

$$
\begin{aligned}
& \sigma_{r r}=\sigma_{\phi \phi}=\frac{3 E_{r} S_{r}}{\left(E_{r}+3\right) S_{r}+4} \varepsilon E_{m} \\
& \sigma_{z z}=\sigma_{r r} \frac{\left(E_{r}+1\right) S_{r}+2}{E_{r} S_{r}+1}
\end{aligned}
$$

siendo $E_{r}=E_{g} / E_{m}$ y $S_{r}=S_{g} / S_{m}$ Error! Bookmark not defined., las relaciones entre módulos de Young y de las secciones, respectivamente, de la capa de vidrio y del núcleo metálico. Se ha tomado el número de Poisson $v=0.3$ en todos los casos.

Teniendo en cuenta las ec. (1) a (4), podemos por último expresar el campo de anisotropía efectivo como:

$$
H_{k}^{e f f}=3 \alpha^{*}\left(\mu_{0} M_{s}(T)\right)^{2}\left(\sigma_{\text {int }}+\sigma_{\text {th.exp. }}(T)\right)+N \mu_{0} M_{s}(T)
$$

donde $\sigma_{\text {int }} \mathrm{y} \sigma_{\text {th.exp }}$ representan las tensiones internas inducidas durante el proceso de fabricación y por la reducción de temperatura respectivamente.

\subsection{Análisis de resultados: influencia de las características geométricas}

Pasamos a continuación a analizar los resultados experimentales antes expuestos. En primer lugar, de la diferencia de los ciclos de histéresis mostrados en las figuras 1a y $1 \mathrm{~b}$ podemos reconocer que la pérdida de perfecta biestabilidad del microhilo corto a temperatura ambiente se debe a la disminución de la imanación con la temperatura y consiguientemente del campo de anisotropía de forma y por tanto del campo de anisotropía efectiva.

En cuanto a los resultados representados en la figura 2, hemos de 
tener en cuenta la diferencia en las tensiones generadas a baja temperatura como consecuencia del diferencial en coeficientes de expansión térmica. Para los microhilos que estamos analizando en esta figura, se pueden tomar los siguiente valores concretos [9]: $\mathrm{E}_{\mathrm{m}}=2 \times 10^{11} \mathrm{~N} / \mathrm{m}^{2}, \mathrm{E}_{\mathrm{g}}$ $=10^{11} \mathrm{~N} / \mathrm{m}^{2}, \alpha_{\mathrm{m}}=8.7 \times 10^{-6} \mathrm{~K}, \alpha_{\mathrm{g}}=3.3 \times 10^{-6} \mathrm{~K}^{-1}$. Con estos valores numéricos, las tensiones inducidas al reducir la temperatura en el rango $\Delta \mathrm{T}$ $=5-300=-295 \mathrm{~K}$ son $\sigma_{\mathrm{rr}}=\sigma_{\phi \phi}=132 \mathrm{MPa}, \mathrm{y} \sigma_{\mathrm{zz}}=388 \mathrm{MPa}$, para el microhilo cuyo diámetro metálico es 3.7 micras. Para el microhilo de 10.8 micras de diámetro metálico se obtienen valores de $\sigma_{\mathrm{rr}}=\sigma_{\phi \phi}=108 \mathrm{MPa}$, y $\sigma_{\mathrm{rr}}=\sigma_{\phi \phi}=132 \mathrm{MPa}$. Es decir, por una parte las tensiones más elevadas son aquellas de carácter de tracción axial por lo que de acuerdo a la ec. (5) dan lugar a un incremento de la anisotropía axial $\left(\sigma_{\text {th.exp. }}=\sigma_{z z}\right)$. Su valor absoluto (388 y $132 \mathrm{MPa}$ respectivamente) son inferiores aunque del mismo orden que las tensiones producidas por el proceso de fabricación que se encuentran en el entorno de $400 \mathrm{MPa}$ [1].

\subsection{Influencia del modo de fijación de la muestra}

A continuación quisiéramos comentar las notables diferencias observadas en los resultados al modificar de modo aparentemente sin mayor importancia el proceso de toma de medidas. Como se ha mencionado antes, las medidas fueron tomadas en un magnetómetro SQUID disponiendo los microhilos de dos formas diferentes. En la primera de ellas, el microhilo se introduce en el interior de un pequeño capilar de diámetro interior entorno a $0.3 \mathrm{~mm}$, y sus extremos son tapados. El microhilo se encuentra por lo tanto libre de tensiones adicionales. De este modo se observan los ciclos que venimos mostrando.

El segundo modo de medida consiste en fijar el microhilo mediante una cinta scotch (habitual en técnicas de medida a bajas temperaturas) al portamuestras. En este caso, y para un microhilo de 2 mm de longitud y composición $\mathrm{Fe}_{72.5} \mathrm{Si}_{12.5} \mathrm{~B}_{15}$, se obtienen los resultados mostrados en la figura 3. Sorprendentemente, ahora la biestabilidad se pierde al bajar la temperatura, y más aún, se induce una fuerte anisotropía transversal cuyo campo de anisotropía alcanza valores entorno a 1 kOe. Este comportamiento sólo se puede explicar si tenemos en cuenta que ahora el microhilo, o mejor, la capa de aislante está fijada y no puede expandirse ni contraerse libremente, de modo que esto resulta en tensiones efectivas de carácter transversal, $\sigma_{\text {trans }}$, inducidas en el núcleo magnético. Si realizamos un cálculo aproximativo para determinar la intensidad de estas tensiones mecánicas inducidas por la bajada de temperatura impidiendo la contracción del vidrio, obtenemos a partir de la ec. (4) un valor $\sigma_{\text {trans }} \approx 1 \times 10^{3} \mathrm{MPa}$.

\section{CONCLUSIONES}

En este trabajo se presenta un estudio acerca de la influencia de las diferentes características geométricas de microhilos sobre la dependencia térmica del proceso de inversión de la imanación, y los correspondientes ciclos de histéresis, en microhilos magnéticos de elevada magnetostricción (entorno a 50 p.p.m.). Las principales conclusiones son:

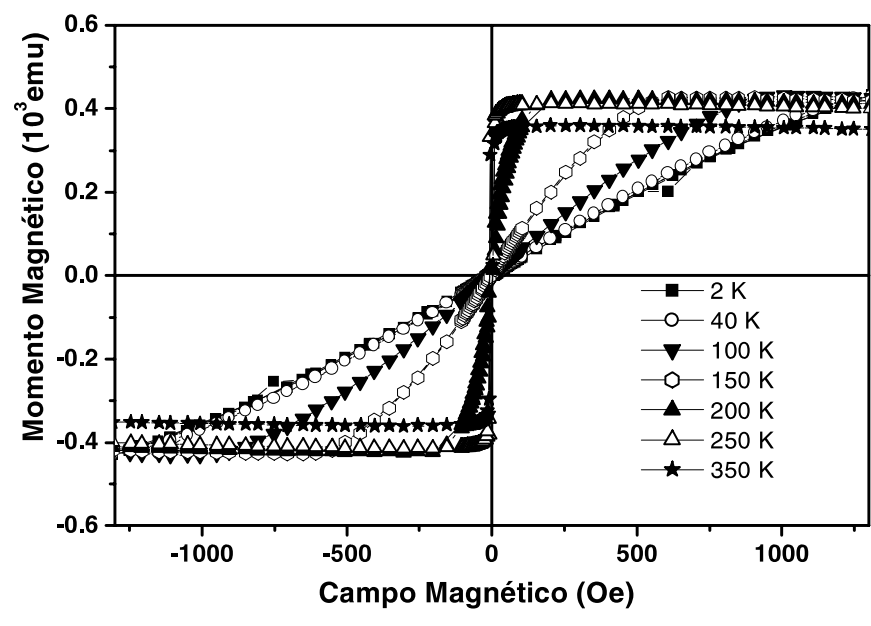

Figura 3.- Dependencia térmica de los ciclos de histéresis para un microhilo de composición $\mathrm{Fe}_{72.5} \mathrm{Si}_{12.5} \mathrm{~B}_{15}$ y longitud $2 \mathrm{~mm}$ fijado al portamuestras con cinta scotch.

i) La longitud del microhilo determina la existencia de comportamiento biestable: microhilos muy cortos (inferiores a $2 \mathrm{~mm}$ ) pueden perder el carácter magnético biestable a temperatura ambiente pero recuperarlo cuando se reduce la temperatura de medida.

ii) La diferencia en coeficientes de expansión térmica de la cubierta protectora de vidrio aislante y el núcleo metálico da lugar a tensiones mecánicas efectivas de tracción axial a bajas temperatura que alcanzan valores entorno a 200-300 MPa, lo que condiciona los parámetros del proceso de inversión de la imanación.

iii) El modo de disponer el microhilo en el portamuestras es de mucha importancia pues la fijacción con cinta scoth puede dar lugar a la generación de tensiones de compresión axiales del orden de hasta $1000 \mathrm{MPa}$.

\section{AGRADECIMIENTOS}

Este trabajo ha sido realizado dentro de los proyectos MAT 20010082-C04-02 (Ministerio de Ciencia y Tecnología).

\section{BIBLIOGRAFÍA}

1. M. Vázquez, A. Zhukov, J. Magn. Magn. Mat. 160 (1996) 223.

2. H. Chiriac, Gh. Pop, A.T. Ovari, Phys. Rev. B 52 (1995) 10104

3. M. Vázquez, D.-X. Chen, IEEE Trans. Magn. 31 (1995) 1229.

4. A.P. Zhukov, M. Vázquez, J. Velázquez, H. Chiriac, V. Larin, J. Magn. Magn. Mat. 151 (1995) 132

5. S. Baranov, V.N. Berzhanski, S. Zotov, V. Kokoz, V. Larin, A. Torcunov, Phys. Met. Metall., 67 (1989) 73.

6. J. Llumá, M. Vázquez, J.M. Hernandez, J.M. Ruiz, J.M. García-Beneytez, A. Zhukov, X.X. Zhang, J. Tejada, J. Magn. Magn. Mat. 196-197 (1999) 821.

7. A.M. Severino, C. Gómez-Polo, P. Marín, M. Vázquez, J. Magn. Magn. Mat. 103 (1992) 117

8. A. Hernando y M. Vázquez, en "Rapidly Solidified Alloys" Ed. H.H. Liebermann (Marcel Dekker Inc., N. York, 1993) pp.553-590

9. M. Vázquez, A.P. Zhukov, K.L. García, K.R. Pirota, A. Ruiz, J.L. Martinez and M. Knobel* RQ'11 (en prensa).

Recibido: 1.2 .03

Aceptado: 30.11 .03 